\title{
Effect of Composition and Formation Conditions on Characteristics of Ammonia Sensor
}

\author{
Olga M. Ivanova, Sergey A. Krutovertsev, Anatoly V. Shevchenko, Alla E. Tarasova \\ JSC "Ecological sensors and systems", 124460, Moscow, Zelenograd, Russia \\ boss@pnc.ru
}

\begin{abstract}
:
The properties of nanostructured SiOx films doped with Dawson-type heteropolycompounds at room temperature in clean air and in air containing ammonia were examined. It was studied adsorption activity of the sensors and found that the films formed at the surface of electrodes have a highly developed surface with nano-size pores. It has been shown that heteropolyanion composition and cation type have a significant influence on a sensor behavior of doped films. The study shows that the conditions of the environment affect the sensors characteristics markedly, which can be improved by choosing of the right procedure of forming and processing.
\end{abstract}

Key words: nanostructured film, Dawson-type polyoxometalate, ammonia

\begin{abstract}
Introduction
Sorption type sensors based on complex composition sorbents, which represent a matrix of basic sorbent activated with various additives, hold a special position among the microelectronic gas sensors. It is wise to select a nanostructure matrix of basic sorbent, into which some adjuvant is incorporated, as a base element of the sensor. The adjuvant helps to promote or provide the formation of analytic signal when the sorbent surface interacts with variable composition gas environment.
\end{abstract}

Nano-size $\mathrm{SiO}_{x}$ films doped with catalytic additives are the most promising materials for gas sensitive layers of microelectronic chemical sorption sensors [1, 2]. Different mechanism may exist to increase a gas sensitivity of activated oxide films depending on additive type and conditions of their formation.

Heteropolycompounds (HPCs) attract a special attention among various substances that can be used as the sensor's components of active layer because of unique combination of their various physical and chemical properties. HPCs are widely used in heterogeneous catalysis and analytical chemistry as catalysts for reductionoxidation processes [3, 4]. HPCs are classified as complex polyoxocompounds. HPC redox properties can be varied in wide ranges by changing a heteropolyanion composition. These compounds can act as reversible reagents in the reactions of ligand exchange and redox transformations, accompanied by changes in current carrier concentration or catalysts of such transformations that can be used in chemical sensors. HPCs can be incorporated directly into the initial matrix of basic sorbent to improve gas sensitivity and selectivity and to reduce intrinsic resistance of sorption nanostructure films.

Modifying the surface of initial sorbent or using the complex composition sorbents is one of the most efficient methods to change the properties of sorption films. Thus, considering that the same base sorbent can be used in various types of sensors, it is possible to specify a general requirement for a method to produce gas-sensitive layers for microelectronic gas sensors. The method must provide a reproducible formation of the thin high-porous nano-size films with complex composition and highly developed specific surface on the substrate at minimum possible temperature. In our opinion, the method of hydrolytic polycondensation of solutions of various organometallic compounds optimally meets the above listed requirements when producing activated $\mathrm{SiO}_{x}$ films. It was selected to form doped sensitive layers of gas sensors as one of the most cost effective and manufacturable methods of thin oxide films production, including ones with complex composition $[6,7]$.

This paper examines an effect of composition and formation conditions on sensitivity of nanostructured silica films doped with Dawsontype heteropolycompounds for ammonia. 


\section{Experimental}

The method of hydrolythic polycondensation from tetraethyl orthosilicate solutions was used for forming nanostructured doped films in this work. Analytical pure reagent TEOS was used to prepare the porous silica gel films. TEOS was thoroughly mixed with alcohol and water. Various quantities of HPCs were used to polymerization of the TEOS to form a sol solution.

Composite films were deposited to $15.0 \times 5.0$ $\mathrm{mm}$ test structures with a system of interdigital metal electrodes formed at their surface (see Fig. 1); active zone of the sensor was $4.0 \times 4.0$ $\mathrm{mm}$.

Different composition heteropolycompounds incorporated into the initial solution. The conditions of film formation varied during the tests: solution composition, temperature, and application modes. Standard gas mixtures (MGPZ, Russia), Dräger test gas ampoules (Dräger, Germany) and Environics - 4000 gasmixture generator (Environics, USA) were used to measure the properties of sensors. Electrical parameters of the sensors have been measured electronically in real-time mode.

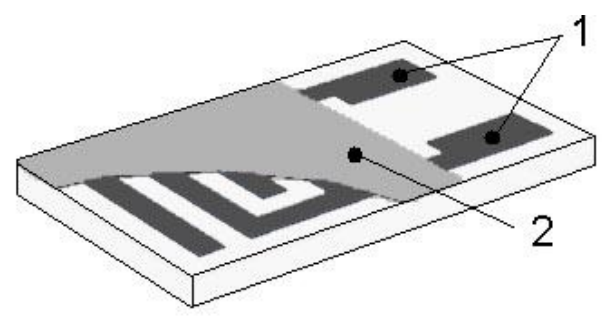

Fig. 1. Test structure for measurements:

1-interdigitated electrodes; 2-nanostructured film.

\section{Results}

The introduction of various synthesized polyoxometalates into $\mathrm{SiO}_{x}$ films results in selectivity and high sensitivity to $\mathrm{NH}_{3}$. The composition of heteropolyanion and cation type markedly influence on sensor behaviour of complex film (see Fig. 2).

Increase of sensitivity and selectivity of such sensors can be received owing to complexing reaction of tested substance wit cationic part of polyoxometalate. This fact results in intramolecular redox reaction which sharply change electrophysical properties of polyoxometalate. Thereby sensitive film's characteristics modify. This process is reversible. It was determined that gas sensitivity increases with rise of molybdenum content in polyoxometalate molecule. Such behaviour is explained by higher reactivity of molybdenum than tungsten.

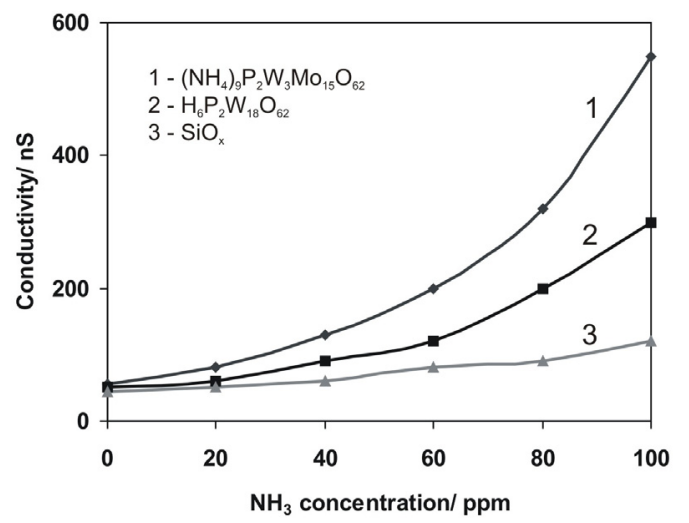

Fig. 2. Typical conductivity dependence of doped $\mathrm{SiO}_{x}$ films on ammonia content in air.

The composition with $30-50 \%$ of the weight content of doping additive in silica matrix enables to obtain a maximum sensitivity to measured gas. It may suppose that the conductivity increase at weight content of modifying admixture less than $50 \%$ connects with increase of charge carriers in the film. Some quantity of extended state protons corresponds to each molecule of polyoxometalate in the film. They can make considerable contribution to proton surface conductivity. It is evident that conductivity decrease of sorption films at weight content of admixture more than $50 \%$ is explained by crystal aggregation of admixture in film volume. The polyoxometalates don't display their catalytic activity at weight content less than 10 $\%$ in the film.

It was established that films have highly developed surface with nanoscale pores. The thickness of the doped $\mathrm{SiO}_{\mathrm{x}}$ layers was $0,2-0,3$ $\mu \mathrm{m}$. The adsorption activity of sensors has been studied using quartz crystal microbalance (see Fig. 3).

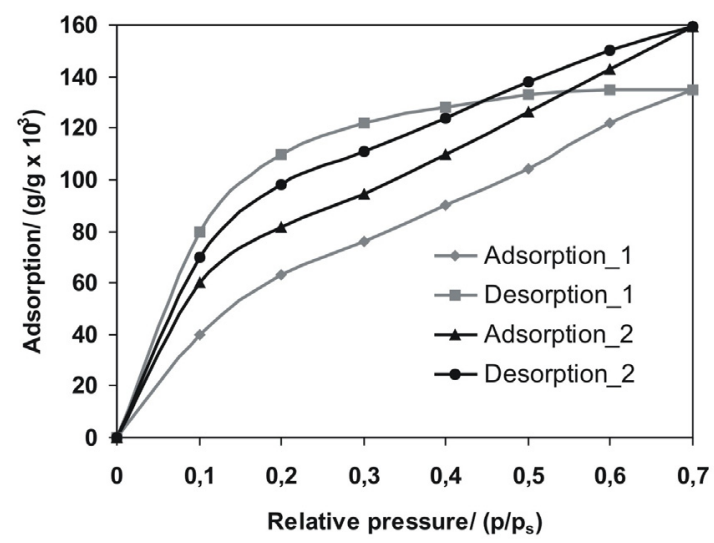

Fig. 3. Adsorption isotherms of ammonia on doped $\mathrm{SiO}_{x}$ films $(298 \mathrm{~K})$ : 1- $\left(\mathrm{NH}_{4}\right)_{9} \mathrm{P}_{2} \mathrm{~W}_{3} \mathrm{Mo}_{15} \mathrm{O}_{62} ; 2$ $\mathrm{H}_{6} \mathrm{P}_{2} W_{18} \mathrm{O}_{62}$.

It has been found that the films had a highly developed surface with nano-size pores. The 
average size of pores was $2-3 \mathrm{~nm}$. The rating value of specific surface measured for sensitive layer is $400-450 \mathrm{~m}^{2} / \mathrm{g}$ for different samples.

The study shows that the conditions of the environment affect markedly the sensors stability, which can be improved by choosing of the right procedure of forming and processing. Forming process of film is long. However introduction of heteropolycompound into film composition makes for film's mass stabilization. The basic rules of films formation remain at that (see Fig.4).

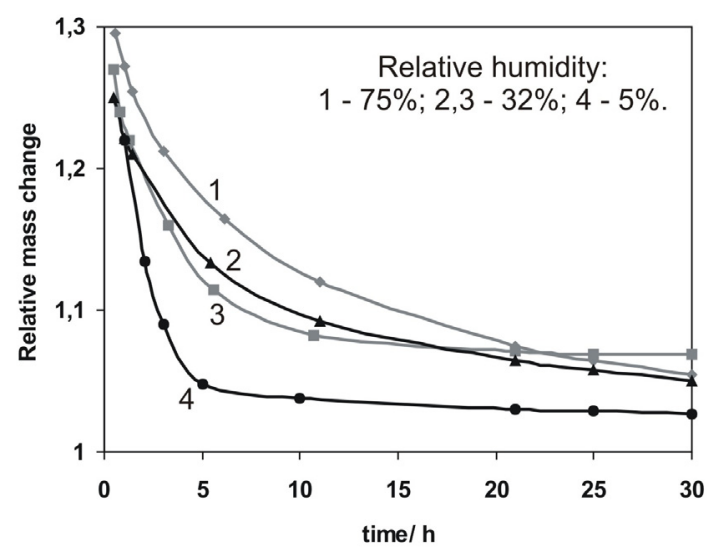

Fig. 4. Dependence of mass change of $\mathrm{SiO}_{x}$ films on time: 1, 2, 4 - undoped silica film; 3 - silica film doped by $\mathrm{H}_{6} \mathrm{P}_{2} \mathrm{~W}_{18} \mathrm{O}_{62}$

The temperature increase stabilization of film (see Fig. 5). So, decreasing of environment humidity and temperature treatment lead to acceleration of hydrolythic polycondensation of solutions and stabilization of sensitive films at right conditions.

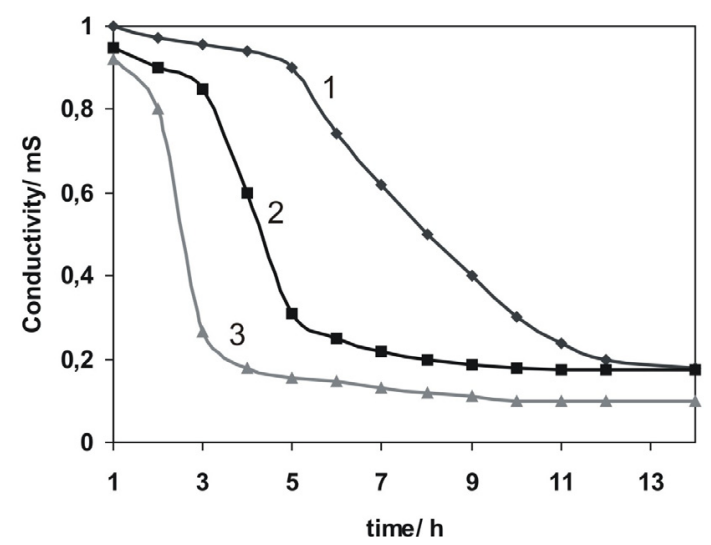

Fig. 5. Conductivity changes for silica film doped by $\left(\mathrm{NH}_{4}\right)_{9} \mathrm{P}_{2} \mathrm{~W}_{3} \mathrm{Mo}_{15} \mathrm{O}_{62}: 298 \mathrm{~K}(1), 353 \mathrm{~K}(2), 423 \mathrm{~K}(3)$.

Threshold sensitivity to $\mathrm{NH}_{3}$ is $0.5-1.0 \mathrm{ppm}$ for $\mathrm{SiO}_{x} /\left(\mathrm{NH}_{4}\right)_{9} \mathrm{P}_{2} \mathrm{~W}_{3} \mathrm{Mo}_{15} \mathrm{O}_{62}$ sensors; range of measured concentrations - 1-100 ppm; and response time - $20 \mathrm{~s}$. Important property of the sensors consists in their ability to operate at room temperature, and this provides indisputable advantages for the development and operation of control instruments on their basis.

\section{Conclusion}

Inorganic oxide films activated with different admixtures are used as the most perspective sensitive layers for sensors working at room temperature. The sensor properties of films can be varied considerably by introducing different activated or modifying admixtures into them and by film formation technologies. The film activation method which consists in adding of admixture to the base sorbate, with the following adsorption of determined gas on the film surface and selective reaction between admixture and gas, allows approaching for selectivity problem solution. Activated nanostructured SiOx films at appropriate conditions can get specified sensor properties.

The polyoxometalates based sensor films may be used for measurements of atmosphere content in ecological monitoring system, in industrial applications, in different medical and biological researches.

\section{References}

[1] Al. Palaniappan, X. Li, F.E.H. Tay, J. Li, X. Su, Cyclodextrin functionalized mesoporous silica films on quartz crystal microbalance for enhanced gas sensing, Sensors and Actuators 119, 220 226 (2006); doi: 10.1016/j.snb.2005.12.015

[2] A. Martucci, D. Buso, M. Guglielmi, L. Zbroniec, N. Koshizaki, M. Post, Optical gas sensing properties of silica film doped with cobalt oxide nanocrystals, Journal Sol-Gel Science Technology 32, 1, 243-246 (2004); doi: $10.1007 / \mathrm{s} 10971-004-5795-3$

[3] Masahiro Sakadane, Eberhard Steckhan, Electrochemical properties of polyoxometalates as electrocatalysts, Chemical Reviews 98, 219237. (1998); doi: 10.1021/cr960403a

[4] N. Fay, E. Dempsey, T. Mccormac, Assembly, electrochemical characterization and electrocatalytic ability of multilayer films based on $\left[\mathrm{Fe}(\mathrm{bpy})^{3}\right]^{2+}$, and the Dawson heteropolyanion $\left[\mathrm{P}_{2} \mathrm{~W}_{18} \mathrm{O}_{62}\right]^{6-}$, Journal of Electroanalytical Chemistry, 574, 2, 359-366 (2005); doi: 10.1016/j.jelechem.2004.07.038

[5] Y. Ma, M. Kanezashi, T. Tsuru, Preparation of organic/inorganic hybrid silica using methyltriethoxysilane and tetraethoxysilane as co-precursors, Journal Sol-Gel Science Technology 53, 93-99 (2010); doi: 10.1007/s10971-009-2061-8

[6] H. Guleryuz, I. Kaus, C. Filiátre, T. Grande, M-A. Einarsrud, Deposition of silica thin films formed by sol-gel method, Journal Sol-Gel Science Technology 54, 249-257 (2010); doi: $10.1007 / \mathrm{s} 10971-010-2190-0$ 